Pacific Journal of Mathematics

CORONA PROBLEM FOR RIEMANN SURFACES OF
PARREAU-WIDOM TYPE 


\title{
CORONA PROBLEM FOR RIEMANN SURFACES OF PARREAU-WIDOM TYPE
}

\section{MitsURU NAKAI}

\begin{abstract}
It is shown that there exists a hyperbolic regular Riemann surface $R$ of Parreau-Widom type that is not dense in the maximal ideal space $\mathscr{M}(R)$ of the Banach algebra $H^{\infty}(R)$ of bounded analytic functions on $R$.
\end{abstract}

It has been close to twenty years since Carleson [1] positively solved the corona problem for the unit disk. Since then various subsequent developments appeared. Among them we are particularly interested in investigations on the corona problem for Riemann surfaces of positive genus. As for the positive direction, Gamelin [2], e.g., proved by using his localization principle that the corona problem can be positively answered for finite Riemann surfaces with analytic borders. As for the negative direction, B. Cole constructed a Riemann surface for which the corona problem is negatively answered (see Gamelin [3]). In connection with these results, it is an interesting and also important problem to single out the class of Riemann surfaces of general genus for which the corona problem is positively settled. One might suspect that the class of Riemann surfaces of Parreau-Widom type falls into this category since the class $H^{\infty}(R)$ of bounded analytic functions on a Riemann surface $R$ of this class is known to share various nice properties with the class $H^{\infty}(D)$ on the unit disk $D$ (cf. Parreau [8], Widom [10, 11], Stanton [9], Hasumi [4, 5], Hayashi [6, 7], etc). The purpose of this paper is to show that the above expectation is unfortunately incorrect.

Consider a hyperbolic Riemann surface $R$ so that there exists the Green's function $g(z, a)$ on $R$ with its pole at any point $a$ in $R$. By the maximum principle for harmonic functions the set $R(\alpha, a)=\{z \in R ; g(z, a)>\alpha\}$ is a subregion of $R$ for any $\alpha>0$ and $a$ in $R$. The surface $R$ is said to be regular if $R(\alpha, a)$ is relatively compact for any $\alpha>0$ and $a$ in $R$. The first Betti number $B(\alpha, a)$ of $R(\alpha, a)$ is the minimum number of generators of the first singular homology group $H_{1}(R(\alpha, a))$ of $R(\alpha, a)$. A hyperbolic Riemann surface $R$ is said to be of Parreau-Widom type if $\int_{0}^{\infty} B(\alpha, a) d \alpha<+$ $\infty$ for one and hence for every $a$ in $R$. We denote by $\mathscr{M}(R)$ the maximal ideal space of $H^{\infty}(R)$ equipped with the Gelfand topology. We may view $\mathscr{M}(R)$ as the space $\{q\}$ of multiplicative linear functionals $q$ on $H^{\infty}(R)$ with $q(1)=1$ equipped with the weak star topo- 
logy since $q \mapsto q^{-1}(0)$ is the bijective homeomorphism between $\{q\}$ and $\mathscr{M}(R)$. A point $z$ in $R$ corresponds to a functional $q_{z}$ in $\mathscr{M}(R)$ defined by $q_{z}(f)=f(z)$ (point evaluation). If $R$ is a hyperbolic Riemann surface of Parreau-Widom type, then this natural mapping $z \mapsto q_{z}$ gives the injective homeomorphism $R \rightarrow \mathscr{M}(R)$ and the image of $R$ under this mapping is open in $\mathscr{M}(R)$ (see Stanton [9]) and therefore we may view $R$ as an open subset of $\mathscr{M}(R)$. The corona problem asks whether $R$ is dense in $\mathscr{M}(R)$ or not. The main result of this paper is the following

THEOREM. There exists a hyperbolic regular Riemann surface $R$ of Parreau-Widom type that is not dense in the maximal ideal space $\mathscr{M}(R)$ of $H^{\infty}(R)$.

The surface $R$ in the above theorem which we will construct is of infinite genus and infinite connectivity. It is obtained from the $\mathrm{B}$. Cole example by making a minor modification. This modification is formulated as proposition in no. 1 , and it is proved in nos. 2-4. The construction of $R$ in the above theorem is carried over in nos. 5-9.

1. Consider a fixed sequence $\left(S_{n}\right)_{1}^{\infty}$ of interiors $S_{n}$ of finite bordered Riemann surfaces $\bar{S}_{n}$ with analytic borders $\partial S_{n}$, two fixed sequences $\left(b_{n}\right)_{1}^{\infty}$ and $\left(c_{n}\right)_{1}^{\infty}$ of real numbers $b_{n}$ and $c_{n}$ with $0<c_{n}<b_{n}$, and a variable sequence $\left(\eta_{n}\right)_{1}^{\infty}$ of real numbers $\eta_{n}$ with $0<\eta_{n} \leqq$ $\min \left(c_{n}, b_{n}-c_{n}\right)$. By using these sequences we will construct a Riemann surface as follows.

Let $X_{n}$ be a rectangular strip $\left\{0 \leqq \operatorname{Re} z \leqq 2,0<\operatorname{Im} z<b_{n}\right\}$ and $X_{n}^{\prime}$ a rectangular strip $X_{n}$ less two vertical slits $\sigma_{n}^{\prime}=\{\operatorname{Re} z=1,0 \leqq$ $\left.\operatorname{Im} z \leqq c_{n}-\eta_{n}\right\}$ and $\sigma_{n}^{\prime \prime}=\left\{\operatorname{Re} z=1, c_{n}+\eta_{n} \leqq \operatorname{Im} z \leqq b_{n}\right\}$, i.e., $X_{n}^{\prime}=$ $X_{n}-\sigma_{n}^{\prime} \cup \sigma_{n}^{\prime \prime}$, for each $n$. Observe that $\tau_{n}=\left\{\operatorname{Re} z=1,\left|\operatorname{Im} z-c_{n}\right|<\right.$ $\eta_{n}$ \} is a cross-cut of $X_{n}^{\prime}$ with the length $2 \eta_{n}$ for each $n$. The left and right vertical sides of $X_{n}^{\prime}$ (and hence of $X_{n}$ ) will be denoted by $\alpha_{n}$ and $\beta_{n}$ respectively.

Weld $X_{n}^{\prime}$ to $S_{n}$ and $S_{n+1}$ by identifying the side $\alpha_{n}$ of $X_{n}^{\prime}$ with an open arc in $\partial S_{n}$ and the side $\beta_{n}$ of $X_{n}^{\prime}$ with an open arc in $\partial S_{n+1}$ for each $n$. The resulting surface $\bigcup_{n=1}^{\infty}\left(S_{n} \cup X_{n}^{\prime}\right)$ will be denoted by

$$
R=R\left(\left(\eta_{n}\right)\right)=R\left(\left(S_{n}\right),\left(X_{n}\right),\left(\eta_{n}\right)\right) .
$$

Here it is aisumed that $\bar{S}_{n} \cap \bar{S}_{m}=\phi(n \neq m), \bar{X}_{n}^{\prime} \cap \bar{X}_{m}^{\prime}=\phi(n \neq m)$, and $\bar{X}_{n}^{\prime} \cap \bar{S}_{k}=\phi(k \neq n, n+1)$ in $R$. By using $X_{n}$ instead of $X_{n}^{\prime}$ we construct the Riemann surface $\bigcup_{n=1}^{\infty}\left(S_{n} \cup X_{n}\right)$ in the same fashion 
as $R\left(\left(S_{n}\right),\left(X_{n}\right),\left(\eta_{n}\right)\right)=\bigcup_{n=1}^{\infty}\left(S_{n} \cup X_{n}^{\prime}\right)$. The resulting surface will be denoted by

$$
R=R\left(\left(S_{n}\right),\left(X_{n}\right)\right) \text {. }
$$

Hence $R\left(\left(S_{n}\right),\left(X_{n}\right),\left(\eta_{n}\right)\right)=R\left(\left(S_{n}\right),\left(X_{n}\right)\right)-\bigcup_{n=1}^{\infty}\left(\sigma_{n}^{\prime} \cup \sigma_{n}^{\prime \prime}\right)$. Clearly the surfaces $R$ in (1) or (2) can be embedded in a larger Riemann surface $W$ such that $W-\bar{R} \neq \phi$. Therefore the surfaces $R$ given by (1) or by (2) are hyperbolic. We will prove the following

Proposition. If the sequence $\left(\eta_{n}\right)_{1}^{\infty}$ converges to zero sufficiently rapidly, then $R=R\left(\left(S_{n}\right),\left(X_{n}\right),\left(\eta_{n}\right)\right)$ is a hyperbolic regular Riemann surface of Parreau-Widom type.

It can happen that $R=R\left(\left(S_{n}\right),\left(X_{n}\right)\right)$ is neither regular nor of Parreau-Widom type. In such a case $R=R\left(\left(S_{n}\right),\left(X_{n}\right),\left(\eta_{n}\right)\right)$ is of the same sort if $\left(\eta_{n}\right)_{1}^{\infty}$ converges to zero not so rapidly. The proof of the proposition will be given in nos. 2-4.

2. We denote by $R_{n}=\bigcup_{k=1}^{n}\left(S_{k} \cup X_{k}^{\prime}\right)-\beta_{n}$ the initial part of $R=R\left(\left(S_{n}\right),\left(X_{n}\right),\left(\eta_{n}\right)\right)$ and by $R_{n}^{\prime}=R-R_{n} \cup \beta_{n}$ the terminal part of $R$. Recall that the first Betti number $B$ of a finite Riemann surface $W$ with border $\partial W$ is given by $B=1-\chi=2 g+m-1$ where $\chi$ is the Euler characteristic of $W, g$ the genus of $W$, and $m$ the number of components of $\partial W$. Let $B_{n}$ be the first Betti number of $R_{n}$. Observe that $B_{n}$ is finite since $R_{n}$ is the interior of the finite bordered surface $\bar{R}_{n}$ and that $B_{n}$ does not depend on the choice of $\left(\eta_{n}\right)$ since $R\left(\left(S_{n}\right),\left(X_{n}\right),\left(\eta_{n}\right)\right)$ are homeomorphic to each other for all choices of $\left(\eta_{n}\right)$. Then fix a sequence $\left(\varepsilon_{n}\right)_{1}^{\infty}$ of positive numbers $\varepsilon_{n}$ such that $\varepsilon_{n}>\varepsilon_{n+1}(n=1,2, \cdots), \lim _{n} \varepsilon_{n}=0$, and $\sum_{n=2}^{\infty} B_{n}\left(\varepsilon_{n-1}-\varepsilon_{n}\right)<$ $+\infty$.

Fix a point $a$ in $S_{1}$ and let $g(z, a)$ be the Green's function of $R=R\left(\left(S_{n}\right),\left(X_{n}\right),\left(\eta_{n}\right)\right)$ with its pole at $a$. We also denote by $\hat{g}(z, a)$ the Green's function of $\hat{R}=R\left(\left(S_{n}\right),\left(X_{n}\right),\left(\eta_{n}^{\prime}\right)\right)$ with $\eta_{n}^{\prime}=\min \left(c_{n}, b_{n}-\right.$ $\left.c_{n}\right)$. Then every $R$ is a subsurface of $\hat{R}$ and therefore $g(z, a) \leqq$ $\hat{g}(z, a)$ on $R$. Choose and fix $M>0$ so large that $\hat{U}=\{z ; \hat{g}(z, a)>M\}$ is contained in $S_{1}$ and simply connected. Then $U=\{z ; g(z, a)>M\}$ is a subset of $\hat{U}$ and also simply connected. Hence the Betti number $B_{0}$ of $U$ is zero.

Let $Y_{n}$ be the part $\left\{1<\operatorname{Re} z \leqq 2,0<\operatorname{Im} z<b_{n}\right\}$ of $X_{n}, \hat{R}_{n}^{\prime}$ the terminal part of $\hat{R}$, and $w_{n}$ the harmonic measure of $\tau_{n}$ with respect to the region $Y_{n} \cup \hat{R}_{n}^{\prime}$. Then

$$
g(z, a) \leqq M w_{n}(z) \quad\left(z \in Y_{n} \cup R_{n}^{\prime}\right)
$$


and, in particular, $\sup _{\beta_{n}} g(\cdot, a) \leqq M \sup _{\beta_{n}} w_{n}$. It is clear that $\lim _{\eta_{n} \rightarrow 0} w_{n}=0$ uniformly on $Y_{n} \cup R_{n}^{\prime}$ less any neighborhood of the left vertical side of $Y_{n}$. We can thus choose a sequence $\left(\eta_{n}\right)_{1}^{\infty}$ converging to zero enough rapidly so that

$$
\sup _{\beta_{n}} g(\cdot, a) \leqq \varepsilon_{n} \quad(n=1,2, \cdots) .
$$

3. We pause here to observe the following. Let $W_{0}$ be a subsurface of a Riemann surface $W$. Consider the first singular homology groups $H_{1}\left(W_{0}\right)$ and $H_{1}(W)$ of $W_{0}$ and $W$, respectively. A cycle $\gamma$ on $W_{0}$ is automatically a cycle on $W$ and this gives a natural group homomorphism $\gamma \mapsto \gamma$ of $H_{1}\left(W_{0}\right)$ to $H_{1}(W)$. Suppose that $W_{0}$ satisfies the condition $(N)$ : Any connected component of $W-W_{0}$ is not compact. Under this condition $(N)$, the above natural homomorphism $H_{1}\left(W_{0}\right) \rightarrow H_{1}(W)$ is injective. To see this, let $\gamma$ be a cycle on $W_{0}$ which is homologous to zero on $W$, i.e., $\gamma \sim 0$ on $W$. We have to show that $\gamma$ is homologous to zero already on $W_{0}$, i.e., $\gamma \sim 0$ on $W_{0}$. Since $\gamma \sim 0$ on $W$ and $\gamma \in H_{1}\left(W_{0}\right)$, we can express $\gamma$ as $\gamma \sim \sum_{j=1}^{l} \partial \sigma_{j}^{2}$ where each $\sigma_{j}^{2}$ is a simplicial 2-simplex on $W$ with $\partial \sigma_{j}^{2} \subset W_{0}$. If $\sigma_{j}^{2} \not \subset W_{0}$ for some $j$, then $\sigma_{j}^{2}$ must contain a component of $W-W_{0}$ since $\partial \sigma_{j}^{2} \subset W_{0}$. This contradicts the condition $(N)$. We have then $\sigma_{j}^{2} \subset W_{0}$ and therefore $\gamma \sim 0$ on $W_{0}$.

4. We have already remarked that $R(\alpha, a)=\{z ; g(z, a)>\alpha\}$ is a region for any $\alpha>0$ as a consequence of the maximum principle for harmonic functions. In view of (3), $R(\alpha, a) \subset R_{n}$ for $\alpha>\varepsilon_{n}$. Since $g(\cdot, a) \leqq \varepsilon_{n}$ on $\partial R_{n}$, we see that $R(\alpha, a)$ is relatively compact for every $\alpha>0$. This proves that $R$ is regular.

Again by the maximum principle, it is readily seen that $R-$ $R(\alpha, a)$ has no compact component. In particular, if $\alpha>\alpha^{\prime}$, then $R(\alpha, a)$ is a subsurface of $R\left(\alpha^{\prime}, a\right)$ satisfying $(N)$ with respect to $R\left(\alpha^{\prime}, a\right)$. Therefore the natural homomorphism $H_{1}(R(\alpha, a)) \rightarrow H_{1}\left(R\left(\alpha^{\prime}\right.\right.$, $a)$ ) is injective and a fortiori the minimum number of generators of $H_{1}(R(\alpha, a)$ ), which is the first Betti number $B(\alpha, a)$ of $R(\alpha, a)$, is less than or equal to that of $H_{1}\left(R\left(\alpha^{\prime}, a\right)\right)$, which is $B\left(\alpha^{\prime}, a\right)$. Therefore we have

$$
B(\alpha, a) \leqq B\left(\alpha^{\prime}, a\right) \quad\left(\alpha \geqq \alpha^{\prime}\right) .
$$

Similarly $R(\alpha, a)$ is a subsurface of $R_{n}$ for $\alpha>\varepsilon_{n}$ with the property $(N)$ and the natural homomorphism $H_{1}(R(\alpha, a)) \rightarrow H_{1}\left(R_{n}\right)$ is injective. Recall that the first Betti number of $R_{n}$ is $B_{n}$ and hence we have

$$
B(\alpha, a) \leqq B_{n} \quad\left(\alpha \geqq \varepsilon_{n}\right) .
$$

For $\alpha \geqq M$, the region $R(\alpha, a)$ is contained in $U$ and simply 
connected. Therefore $B(\alpha, a)=0$ for $\alpha \geqq M$ and hence $\int_{M}^{\infty} B(\alpha, a) d \alpha=$ 0 . By (5) we see that $\int_{\varepsilon_{1}}^{M} B(\alpha, a) d \alpha \leqq B_{1}\left(M-\varepsilon_{1}\right)$. Again by (5), $\int_{\varepsilon_{n}}^{\varepsilon_{n-1}} B(\alpha, a) d \alpha \leqq B_{n}\left(\varepsilon_{n-1}-\varepsilon_{n}\right)^{\varepsilon_{1}}$ for every $n \geqq 2$. Hence we have

$$
\int_{0}^{\infty} B(\alpha, a) d \alpha \leqq B_{1}\left(M-\varepsilon_{1}\right)+\sum_{n=2}^{\infty} B_{n}\left(\varepsilon_{n-1}-\varepsilon_{n}\right)<+\infty,
$$

which proves that $R=R\left(\left(S_{n}\right),\left(X_{n}\right),\left(\eta_{n}\right)\right)$ is of Parreau-Widom type.

5. We proceed to the construction of $R$ in our theorem. For the purpose we will briefly describe here how the $B$. Cole example $W$ is constructed that is not dense in the maximal ideal space $\mathscr{M}(W)$ of $H^{\infty}(W)$. The construction is done based upon two crucial steps. The first is the following Existence of a malformed finite surface: Let $\delta$ be an arbitrary real number with $0<\delta<1$ and $m$ an arbitrary positive integer. Then there exists a finite bordered Riemann surface $\bar{W}_{m}$ with its interior $W_{m}$ and with an analytic border $\partial W_{m}$ and a pair $f_{m}, g_{m}$ of functions in $H^{\infty}\left(W_{m}\right)$ with $\left|f_{m}\right|$, $\left|g_{m}\right| \leqq 1$ and $\left|f_{m}\right|+\left|g_{m}\right| \geqq \delta$ on $W_{m}$ such that whenever $f_{m} \phi+g_{m} \psi=$ 1 is satisfied on $W_{m}$ for a pair $\phi$ and $\psi$ of functions in $H^{\circ}\left(W_{m}\right)$, we have $\sup _{W_{m}}|\phi|+\sup _{W_{m}}|\psi| \geqq m$. For its proof see Gamelin [3, pp. 47-49].

6. Another important device for the construction is the following Approximation theorem which is easily deduced by a standard successive approximation procedure from the Bishop generalization to Riemann surfaces of the Mergelyan approximation theorem: Let $\left(K_{n}\right)_{1}^{\infty}$ be a sequence of compact subsets $K_{n}$ of a Riemann surface $R$ such that $K_{n} \cap K_{m}=\phi(n \neq m), \gamma_{n}$ a curve in $R-\bigcup_{k=1}^{\infty} K_{k}$ except its end points connecting a boundary point of $K_{n}$ to a boundary point in $K_{n+1}$ such that $\gamma_{n} \cap \gamma_{m}=\phi(n \neq m)$. Assume that $R-F$ has no relatively compact component where $F=\bigcup_{n=1}^{\infty}\left(K_{n} \cup \gamma_{n}\right)$. To each function $\phi$ continuous on $F$ and analytic in the interior of $F$ and each positive number $\varepsilon$ there exists an analytic function $\Phi$ on $R$ such that $\sup _{K_{n} \cup r_{n}}|\Phi-\phi|<\varepsilon / n(n=1,2, \cdots)$.

7. Let $W_{m}$ be as in no. 5 for each $m$ and $Z_{m}$ be a finite Riemann surface obtained from $W_{m}$ by attaching an annulus to each boundary component of $W_{m}$. By using a sequence $\left(L_{m}\right)_{1}^{\infty}$ of rectangular strips $L_{m}$ we construct a Riemann surface $R\left(\left(Z_{m}\right),\left(L_{m}\right)\right)$ defined in (2). Let $\gamma_{m}$ be a curve in $R\left(\left(Z_{n}\right),\left(L_{n}\right)\right)-\bigcup_{1}^{\infty} \bar{W}_{n}$ except its end points connecting a boundary point of $\bar{W}_{m}$ and a boundary point of $\bar{W}_{m+1}$ for each $m$ such that $\gamma_{n} \cap \gamma_{m}=\phi(n \neq m)$. Then $F=\bigcup_{1}^{\infty}\left(\bar{W}_{n} \cup \gamma_{n}\right)$ 
is qualified to be an $F$ in no. 6 with respect to the Riemann surface $R\left(\left(Z_{n}\right),\left(L_{n}\right)\right)$. Consider continuous functions $f_{0}$ and $g_{0}$ on $F$ such that $f_{0}\left|W_{m}=f_{m}, g_{0}\right| W_{m}=g_{m}(m=1,2 \cdots),\left|f_{0}\right|,\left|g_{0}\right| \leqq 1$ and $\left|f_{0}\right|+$ $\left|g_{0}\right| \geqq \delta$ on $F$. By no. 6 there exist analytic functions $f$ and $g$ on $R\left(\left(Z_{n}\right),\left(L_{n}\right)\right)$ such that

$$
\sup _{\bar{W}_{m} \cup r_{m}}\left|f-f_{0}\right|+\sup _{\bar{W}_{m} \cup r_{m}}\left|g-g_{0}\right|<\delta / 4 m \quad(m=1,2, \cdots) .
$$

8. Let $W$ be a connected neighborhood of $F$ in $R\left(\left(Z_{n}\right),\left(L_{n}\right)\right)$ such that $|f|,|g| \leqq 2,|f|+|g| \geqq \delta / 2$ on $W$. The surface $W$ is the B. Cole example (see Gamelin [3, pp. 49-52]). For each $m$, let $S_{m}$ be a finite surface with an analytic border $\partial S_{m}$ such that $\bar{W}_{m} \subset$ $S_{m} \subset \bar{S}_{m} \subset W \cap Z_{m}$ and $Y_{m}$ a rectangular strip $\{0 \leqq \operatorname{Re} z \leqq 2,0<$ $\left.\operatorname{Im} z<b_{m}\right\}$ in the sense of conformal equivalence such that the left side of $Y_{m}$ is a part of $\partial S_{m}$, the right side of $Y_{m}$ is a part of $\partial S_{m+1}, Y_{m} \cap\left(\bigcup_{1}^{\infty} S_{n}\right)=\phi, \quad \bigcup_{1}^{\infty}\left(\bar{S}_{m} \cup \bar{Y}_{m}\right) \subset W$, and $\bigcup_{1}^{\infty}\left(S_{n} \cup Y_{n}\right) \supset F$. Consider the surface $R\left(\left(S_{n}\right),\left(Y_{n}\right)\right)$ defined in (2), which may also be called the $B$. Cole example since it is a neighborhood of $F$ contained in $W$. Let $\sigma_{n}^{\prime}$ and $\sigma_{n}^{\prime \prime}$ be as in no. 1 with $c_{n}=b_{n} / 2$, for example. We finally consider

$$
R=R\left(\left(S_{n}\right),\left(Y_{n}\right),\left(\eta_{n}\right)\right)=R\left(\left(S_{n}\right),\left(Y_{n}\right)\right)-\bigcup_{n=1}^{\infty}\left(\sigma_{n}^{\prime} \cup \sigma_{n}^{\prime \prime}\right)
$$

defined in (1). By proposition in no. $1, R=R\left(\left(S_{n}\right),\left(Y_{n}\right),\left(\eta_{n}\right)\right)$ can be made to a hyperbolic regular Riemann surface of Parreau-Widom type if the sequence $\left(\eta_{n}\right)_{1}^{\infty}$ is so chosen that it converges to zero sufficiently rapidly.

9. Since the surface $R$ given by (7) now so made that it is of Parreau-Widom type, it has many nice properties concerning the class $H^{\infty}(R)$. For example, $H^{\infty}(R)$ separates points in $R$ and the natural injection $R \rightarrow \mathscr{M}(R)$ of $R$ into the maximal ideal space $\mathscr{M}(R)$ of $H^{\circ}(R)$ is bicontinuous and the image in $\mathscr{M}(R)$, identified with $R$, is open in $\mathscr{M}(R)$ (Stanton [9]). The proof of our theorem is over if we show that $R$ is not dense in $\mathscr{M}(R)$.

Observe that functions $f$ and $g$ in no. 3 may be viewed as in $H^{\infty}(R)$ and, by (6), satisfy $|f|,|g| \leqq 2,|f|+|g| \geqq \delta / 2$ on $R$, and

$$
\sup _{\bar{W}_{m}}\left|f-f_{m}\right|+\sup _{\bar{W}_{m}}\left|g-g_{m}\right|<1 / m \quad(m=1,2, \cdots),
$$

where $\bigcup_{1}^{\infty} \bar{W}_{n} \subset R$. Suppose that the indefinite equation $f \phi+g \psi=1$ on $R$ has solutions $\phi$ and $\psi$ in $H^{\infty}(R)$. Set $f_{m} \phi+g_{m} \psi=\lambda_{m}$ on $W_{m}$. Observe that $\sup _{\bar{W}_{m}}\left|1-\lambda_{m}\right|<\left(\sup _{\bar{W}_{m}}|\phi|+\sup _{\bar{W}_{m}}|\psi|\right) / m$ as a consequence of (8). Therefore $f_{m}\left(\phi / \lambda_{m}\right)+g_{m}\left(\psi / \lambda_{m}\right)=1$ on $W_{m}$ with $\phi / \lambda_{m}$ and $\psi / \lambda_{m}$ 
in $H^{\infty}\left(W_{m}\right)$. By no. 5, we then have $\sup _{\bar{W}_{m}}|\phi| \lambda_{m}\left|+\sup _{\bar{w}_{m}}\right| \psi / \lambda_{m} \mid \geqq m$ $(m=1,2, \cdots)$, which contradicts $\sup _{\bar{N}_{m}}\left|1-\lambda_{m}\right| \rightarrow 0 \quad(m \rightarrow \infty)$. This shows that there exists a maximal ideal $M_{0}$ in $\mathscr{C}(R)$ containing $f$ and $g$. Then $f\left(M_{0}\right)=g\left(M_{0}\right)=0$ and the assumption inf $(|f|+|g|) \geqq$ $\delta / 2>0$ imply that $M_{0}$ is not in the closure of $R$ in $\mathscr{L}(R)$ so that $R$ is not dense in $\mathscr{C}(R)$.

\section{REFERENCES}

1. L. Carleson, Interpolation by bounded analytic functions and corona problem, Ann. of Math., 76 (1962), 542-559.

2. T. Gamelin, Localization of the corona problem, Pacific J. Math., 34 (1970), 73-81.

3. — Uniform Algebras and .Jensen Measures, London Math. Soc. Lecture Notes Series 32, Cambridge Univ. Press, 1978.

4. M. Hasumi, Invariant subspaces on open Riemann surfaces, Ann. Inst. Fourier, 24 (1974), 241-286.

5. - Invariant subspaces on open Riemann surfaces II, Ann. Inst. Fourier, 26 (1976), 273-299.

6. M. Hayashi, Hardy classes on Riemann surfaces, Doctoral Dissertation at University of California, Los Angeles, 1979.

7. - Invariant subspaces on Riemann surfaces of Parreau-Widom type, Preprint.

8. M. Parreau. Théorème de Fatou et problème de Dirichlet pour les lignes de Green de certain surfaces de Riemann, Ann. Acad. Sci. Fenn., Ser. A.I. 250/255 (1958), 8pp.

9. C. Stanton, Bounded analytic functions on a class of open Riemann surfaces, Pacific J. Math., 59 (1975), 557-565.

10. H. Widom, The maximum principle for multiple-valued analytic functions, Acta Math., 126 (1971), 63-82.

11. - $\mathscr{K}_{p}$ sections of vector bundles over Riemann surfaces, Ann of Math., 94 (1971), 305-324.

Received January 13, 1981. This work was partly supported by Grant-in-Aid for Scientific Research, No. 454027, Japanese Ministry of Education, Science and Culture.

Nagoya Institute of Technology

Gokiso, Showa, Nagoya 466

JAPAN 



\section{PACIFIC JOURNAL OF MATHEMATICS}

\section{EDITORS}

DONALD BABBITT (Managing Editor)

University of California

Los Angeles, California 90024

Hugo RossI

University of Utah

Salt Lake City, UT 84112

C. C. Moore and Arthur Agus

University of California

Berkeley, CA 94720
J. DugundJI

Department of Mathematics University of Southern California Los Angeles, California 90007

R. FInN and J. Milgram Stanford University Stanford, California 94305

ASSOCIATE EDITORS
R. ARNES
E. F. BeCKenbach
B. H. NeumanN
F. WOLF
K. YoSHIDA

\section{SUPPORTING INSTITUTIONS}

UNIVERSITY OF ARIZONA

UNIVERSITY OF BRITISH COLUMBIA

CALIFORNIA INSTITUTE OF TECHNOLOGY

UNIVERSITY OF CALIFORNIA

MONTANA STATE UNIVERSITY

UNIVERSITY OF NEVADA, RENO

NEW MEXICO STATE UNIVERSITY

OREGON STATE UNIVERSITY
UNIVERSITY OF OREGON

UNIVERSITY OF SOUTHERN CALIFORNIA

STANFORD UNIVERSITY

UNIVERSITY OF HAWAII

UNIVERSITY OF TOKYO

UNIVERSITY OF UTAH

WASHINGTON STATE UNIVERSITY

UNIVERSITY OF WASHINGTON 


\section{Pacific Journal of Mathematics}

\section{Vol. 103, No. $1 \quad$ March, 1982}

Abdul Aziz, On the zeros of composite polynomials ..................

Salomon Benzaquen and Enrique M. Cabaña, The expected measure of the level sets of a regular stationary Gaussian process $\ldots \ldots \ldots \ldots \ldots$

Claudio D’Antoni, Roberto Longo and László Zsidó, A spectral mapping theorem for locally compact groups of operators $\ldots \ldots \ldots \ldots \ldots \ldots \ldots 17$

Ronald Dotzel, Semifree finite group actions on homotopy spheres ........ 25

Daniel H. Gottlieb, The Lefschetz number and Borsuk-Ulam theorems . . . . . 29

Shui-Hung Hou, On property $(Q)$ and other semicontinuity properties of

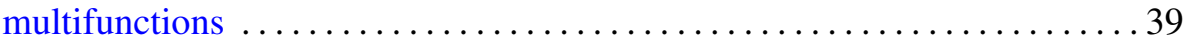

Kevin Mor McCrimmon, Compatible Peirce decompositions of Jordan triple systems

Mitsuru Nakai, Corona problem for Riemann surfaces of Parreau-Widom

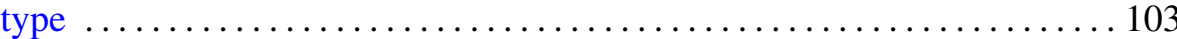

Jack Ray Porter and R. Grant Woods, Extensions of Hausdorff spaces . . . . 111

Milton Rosenberg, Quasi-isometric dilations of operator-valued measures

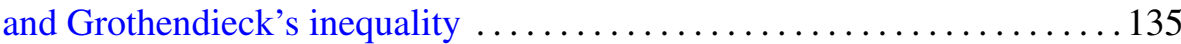

Joseph L. Taylor, A bigger Brauer group $\ldots \ldots \ldots \ldots \ldots \ldots \ldots \ldots \ldots \ldots$

Thomas Vogel, Symmetric unbounded liquid bridges . . . . . . . . . . . 205

Steve Wright, The splitting of operator algebras. II ............... 243 